\title{
Performance Optimization of GaAs-based vertical-cavity surface-emitting transistor-lasers
}

\author{
Yu Xiang, Carl Reuterskiöld-Hedlund, Xingang Yu, Chen Yang, Thomas Zabel, Mattias Hammar, and \\ Muhammad Nadeem Akram
}

\begin{abstract}
We report on the optimization of pnp-type verticalcavity surface-emitting transistor-lasers based on the fusion between an AlGaAs/GaAs heterojunction bipolar transistor and an InGaAs/GaAs VCSEL using an epitaxial regrowth process. It is shown how a proper design of the base region can extend the transistor active range of operation well beyond lasing threshold, thereby resulting in typical transistor laser operational characteristics including $\mathrm{mW}$-range output power, mA-range base threshold current, record-low power dissipation under laser operation, and continuous-wave operation up to at least $60^{\circ} \mathrm{C}$. A pronounced breakdown in the collector current characteristics in the limit of high base current and/or emitter-collector voltage accompanied by a quenching of the optical output power is interpreted as being related to quantum well band-filling.
\end{abstract}

Index Terms-Semiconductor lasers, surface emitting lasers, transistor lasers

\section{INTRODUCTION}

$\mathrm{B}$ ASED on the homogeneous integration of a semiconductor diode laser and a high-speed heterojunction bipolar transistor (HBT), the transistor laser has been introduced as a novel means of extending the functionality and performance of the semiconductor diode laser, displaying a multifold of potential advantages such as improved modulation and noise properties, reduced power consumption, and novel functionality due to the three-terminal approach [1]. Especially the vertical-cavity surface-emitting transistor laser (T-VCSEL) would be very attractive for a range of applications. Combining the functionality and performance advantages of transistor lasers with the inherent advantages of VCSELs, it has been suggested as a potential cost- and power-efficient emitter with greatly extended modulation bandwidth as compared to conventional diode VCSELs [2]. The first experimental demonstration of a T-

Manuscript received October x, 2014; revised October y, 2014; accepted October xx, 2014. Date of publication October yy, 2014; date of current version November zz, 2014. This work was supported by the Swedish Research Council under Grant 2010-4386.

Y. Xiang, C. Reuterskiöld-Hedlund, X. Yu, C. Yang, T. Zabel, and M Hammar are with the School of Information and Communication Technology at KTH-Royal Institute of Technology, Electrum 229, S-164 40 Kista, Sweden (e-mail: hammar@kth.se).

M.N. Akram is with the University College Buskerud and VestFold, N3103 Tønsberg, Norway (e-mail: Muhammad.N.Akram@hbv.no).

Color versions of one or more of the figures in this letter are available online at http://ieeexplore.ieee.org.

Digital Object Identifier
VCSEL was reported by Wu, Feng and Holonyak in 2012 [3]. Studying the static performance of these npn-type T-VCSELs, they showed features such as gain-compression due to the onset of stimulated emission and voltage-controlled operation [4]. From an optimized device structure with an asymmetric oxidation-confined current injection [5] and a hybrid semiconductor/dielectric top DBR they also demonstrated how the device efficiency could be improved for reduced power dissipation [2]. However, these T-VCSELs were only designed for low-temperature operation $\left(-75^{\circ} \mathrm{C}\right)$ with an output power below $100 \mu \mathrm{W}$ [2].

We previously reported lasing from a pnp-type T-VCSEL [6], including $\mathrm{mW}$-range output power and high-temperature operation up to $50^{\circ} \mathrm{C}$. However, the light-current-voltage (LIV) characteristics of these devices did not fully match the requirements for a transistor laser. In specific, a sub-threshold sharp kink in the collector-versus-base current characteristics, reducing the differential gain to zero, indicated that the transistor went into saturation before lasing. As discussed in more detail elsewhere [7], this is due to a lateral potential variation and a gradual turn-on of the base-collector junction. While the transistor is in its active mode of operation locally in the interior of the device, it globally operates in the saturation regime as measured on the electrical contacts. This is obviously an undesirable situation that obstructs the potentially superior properties of a transistor laser. It is therefore of importance to optimize the design so that the transistor active mode of operation is extended well beyond lasing threshold.

In the present work, we demonstrate T-VCSELs designed for an improved internal biasing. By an extension of the base thickness it is shown possible to significantly delay the onset of saturation, thereby sustaining full transistor laser performance over an extended range of base current beyond threshold. The devices work over a large temperature range up to at least $60^{\circ} \mathrm{C}$ with a room-temperature output power close to $1 \mathrm{~mW}$. The LIV characteristics display a rich variety of features related to the interaction between electrical field, carrier dynamics and optical field. In the limit of high base current and/or collector-emitter voltage we observe a breakdown in the collector current-characteristics and a corresponding quenching of the emitted light intensity similar to the observations made by $\mathrm{Wu}$ et al. for the case of npn-type T-VCSELs [2,4], who attributed it to intracavity photonassisted band-to-band tunneling and proposed it as a means for 
voltage-modulation [4]. However, based on the present data we argue that a photon-assisted absorption process is unlikely as the main mechanism behind the breakdown in the LIV characteristics and that this rather is due to quantum-well band-filling.

\section{DEVICE DESIGN AND FABRICATION}

The device structure, schematically illustrated in Fig. 1, has been described in detail elsewhere [6,7]. In short it consists of an undoped 36.5-period AlGaAs/GaAs bottom DBR, a pdoped (Zn: $2 \cdot 10^{17} \mathrm{~cm}^{-3}$ ) GaAs collector region, an n-doped (Si: $6 \cdot 10^{18} \mathrm{~cm}^{-3}$ ) base region also comprising a triple quantum-well (QW) active region (gain maximum at $965 \mathrm{~nm}$ ), a p-doped AlGaAs emitter (Zn: $2 \cdot 10^{18} \mathrm{~cm}^{-3}$ ), and a p-doped GaAs current-spreading layer with an n-type current-confinement layer formed by selective etching and a two-step epitaxial regrowth leading to square-shaped openings of various sizes (2-10 $\mu \mathrm{m}$ side lengths) defining the active region. A fourperiod dielectric $\alpha-\mathrm{Si} / \mathrm{SiO}_{2} \mathrm{DBR}$ is used as out-coupling mirror. The epitaxial layer structures include three different design variations with increasing base thickness regions. The n-type base doping is distributed over two different regions starting $15 \mathrm{~nm}$ above and below the MQW active region. The region above the active layer has a thickness of $240 \mathrm{~nm}$ for all devices whereas the lower region has an extension of 10 (similar to Refs. 6 and 7), 100 or $200 \mathrm{~nm}$, below referred to as 10-, 100-, and 200-nm devices. This is compensated by a reduction of the collector region so that all devices have a 6.5$\lambda$ cavity with an emission wavelength of approximately 1000 $\mathrm{nm}$. As compared to our previous design [6,7], the present devices also have a lower Al-content in the AlGaAs emitter region (38 instead of $88 \%$ ).

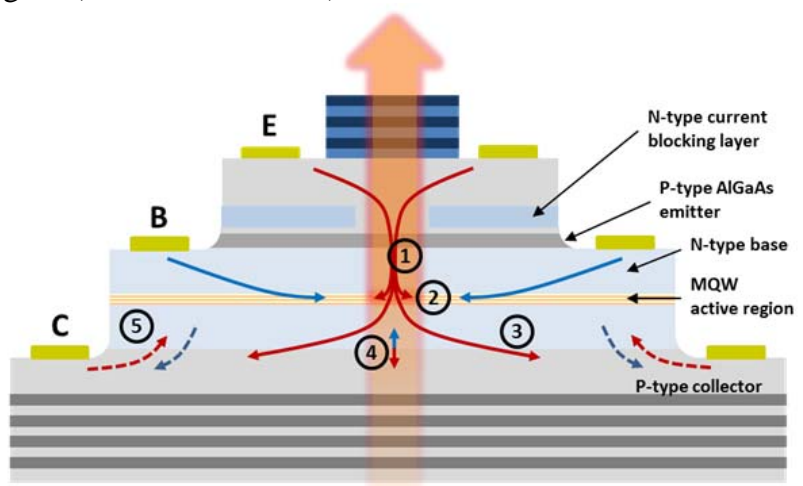

Fig. 1. Schematic illustration of the fabricated T-VCSELs. E, B and C indicate the emitter, base and collector contacts. Blue and red colors indicate electron and hole currents, respectively. The different current contributions are: 1) Hole injection over the forward-biased emitter-base junction $\left(I_{E h}\right)$; 2) Base current $\left(I_{B}\right)$; 3) Part of the emitter hole current that is swept into the collector; 4) Majority-current components due to tunneling of electron-hole pairs at the base-collector junction; and 5) Electron and hole injection over the basecollector junction (only effective in the transistor saturation regime).

\section{RESULTS AND DISCUSSION}

As discussed in more detail elsewhere, an extended base region thickness changes the potential distribution in the device so that the base-collector junction remains reverse

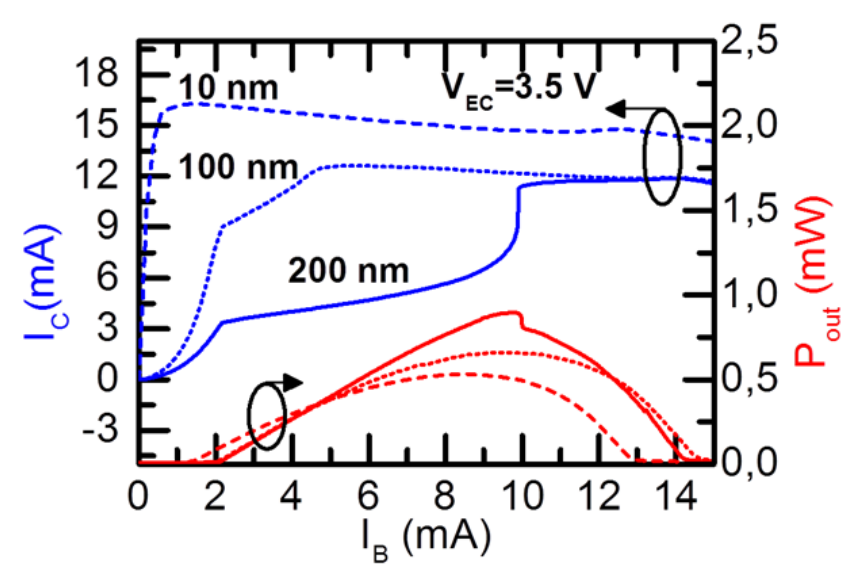

Fig. 2. Optical output power and collector current as function of base current measured at room temperature for the three different base designs, labeled 10 (solid line), 100 (dashed line) and $200 \mathrm{~nm}$ (dashed-dotted line). The device size is $10 \mu \mathrm{m}$ and the applied collector-emitter voltage is $3.5 \mathrm{~V}$.

biased as the base current is increased above threshold [8]. This is a combined effect of reduced base resistance and decreasing current gain with increasing base thickness, leading to reduced $V_{E B}$ and voltage drop along the collector. In Fig. 2, we compare the dependencies of the collector current $\left(I_{C}\right)$ and optical output power $\left(P_{\text {out }}\right)$ on the base current $\left(I_{B}\right)$ for a specific emitter-collector voltage $\left(V_{E C}\right)$ of $3.5 \mathrm{~V}$ for the three different designs, which provide distinctly different LIV characteristics. In case of the 10 -nm device, there is a sharp kink in $I_{C}$ already at $I_{B} \approx 1 \mathrm{~mA}$ and the differential current gain ( $\beta=d I_{C} / d I_{B}$ ) becomes negative before onset of lasing which indicates that the transistor is already in saturation $\left(I_{B s a t}<\right.$ $I_{B t h}=1.4 \mathrm{~mA}$ ). The situation is quite different for the $100-\mathrm{nm}$ device. Here we observe that the first kink coincides with the onset of lasing $\left(I_{B t h}=2 \mathrm{~mA}\right)$. This corresponds to a reduction of $\beta$ but different from the 10 -nm device it is still positive and continues to increase with increasing $I_{B}$ until $I_{C}$ reaches a second kink at $I_{B}=4.5 \mathrm{~mA}$ where $\beta$ turns negative. We interpret the first kink as being due to a sudden increase in the base recombination current due to the onset of stimulated emission [1] and the second kink as due to transistor saturation. For the 200-nm device, the occurrence of the second kink is delayed to $I_{B}=10 \mathrm{~mA}$. Furthermore, we here observe a steep breakdown-like increase in $I_{C}$ at $I_{B}=10 \mathrm{~mA}$ after which the optical output power declines. This switching behavior in the output characteristics will be further addressed below. Consequently, the device is in the active mode of transistor operation up to $4.5 \mathrm{~mA}$ or $10 \mathrm{~mA}$ for the 100 - and 200-nm devices, respectively. This clearly demonstrates the effect of increasing the base region thickness in the T-VCSEL design [8].

Next, we focus on the 200-nm design in some more detail. Figure 3 shows the dependencies of $P_{\text {out }}, I_{C}$ and $V_{E B}$ on $I_{B}$ for a $10-\mu \mathrm{m}$ device at different values of $V_{E C}$. Several operational regimes (indicated by numbers I-IV) are encountered as the base current is increased. Below threshold $I_{C}$ is increasing superlinearly with increasing $I_{B}$ (Region $\mathrm{I}$ ), corresponding to an increasing current gain limited by recombination loss of injected holes in the base (contribution 2 in Fig. 1), mainly from spontaneous recombination in the QWs. The situation is 


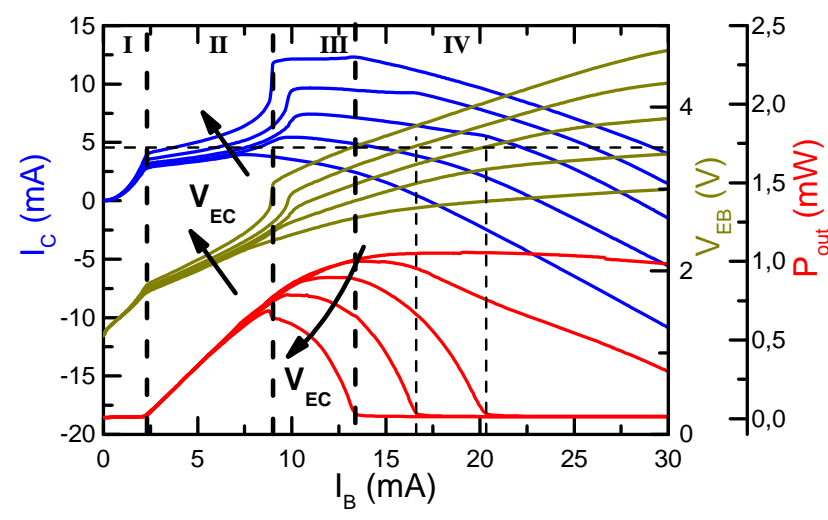

Fig. 3. Collector current, base-emitter voltage and optical output power as function of base current for different emitter-collector voltages between 1.5 and $3.5 \mathrm{~V}$ as indicated. The data is taken at room temperature for a $200-\mathrm{nm}$ device design with an aperture size of $10 \mu \mathrm{m}$. The dashed lines are included as guides to the eye. The regions I - IV applies to $V_{E C}=3.5 \mathrm{~V}$.

drastically changed when the base current reaches lasing threshold (Region II), as manifested by well-pronounced kinks in $P_{\text {out }}, I_{C}$ and $V_{E B}$. This corresponds to the onset of stimulated recombination, in turn leading to a significant increase in the recombination current and thereby a pronounced gain compression. This additional contribution to $I_{B}$ also leads to a flattening of $V_{E B}$. As $I_{B}$ is further increased (still in Region II), $I_{C}$ is gradually increasing at an increasing rate with a breakdown-like behavior (at the boundary between Regions II and III) most pronounced for $V_{E C} \geq 3 \mathrm{~V}$, eventually forcing the transistor into saturation.

A similar breakdown in $I_{C}$ has previously been observed on npn-type T-VCSELs by Wu et al. [2,4], who attributed it to a photon reabsorption process (Franz-Keldysh effect) in the base-collector junction that adds carriers to $I_{C}$ and also acts as a source of resupply of majority carriers to the base $[4,9]$. However, Franz-Keldysh absorption would here not be expected to increase beyond the breakdown in $I_{C}$ (at $I_{B}=I_{B, B D}$ ) since any further increase in $I_{B}$ (corresponding to an increase in $V_{E B}$ and thereby a decrease in $V_{B C}=V_{E C}-V_{E B}$ ) will decrease rather than increase the band bending in the base-collector junction. The step-like drop in intensity at $I_{B, B D}$ (mainly observed for $V_{E C}=3.5 \mathrm{~V}$ ) presumably reflects a temperature rise from the run-off in $I_{C}$ and $V_{E B}$ and is followed by a slower decline throughout Region III.

A striking observation is that total quenching of the light always occurs at a specific $V_{E B Q} \approx 3.5 \mathrm{~V}$ at room temperature regardless of aperture size or $V_{E C}$ (see dashed lines in Fig. 3), which implies that the decline in optical intensity rather is related to the band structure and carrier distribution in the base region. A possible interpretation is that the increase in $V_{E B}$ will alter the supply of carriers to the MQW active region. The injected excess hole concentration will be subject to lateral diffusion resulting in carrier recombination remote from the central part of the device and the lasing mode, eventually leading to insufficient supply of electrons to maintain threshold carrier density.

Region III corresponds to the onset of transistor saturation. Due to the potential drop along the collector, the basecollector junction will gradually turn on to be locally forward biased and thereby supporting a negative contribution to $I_{C}$ (current contribution 5 in Fig. 1) [7]. This implies that in Region III there are three competing contributions to $I_{C}$ : A positive contribution due to photon-assisted carrier transport (evident from the kink in $I_{C}$ at $V_{E B Q}$ ), possibly according to the Franz-Keldysh process described above (contribution 4); a positive contribution stemming from the partly reverse-biased base-collector junction (contribution 3); and a negative contribution from the partly forward-biased base-collector junction (contribution 5). With increasing $I_{B}$ through Region $\mathrm{IV}$, this latter contribution will increase until it eventually dominates and results in an overall negative $I_{C}$.

The electrical and optical collector diagrams $\left(I_{C}\right.$ and $P_{\text {out }}$ as function of $V_{E C}$ for different values of $I_{B}$ ) in Fig. 4 further shows the strong relationship between $I_{C}$ and $P_{\text {out }}$ where the breakdown in $I_{C}$ perfectly lines up with a quenching of the light intensity for each value of $I_{B}$ until saturation. It may be noted that for increasing $I_{B}$ the breakdown occurs at reduced $V_{E C}$ which is similar to the observation in Fig. 3 where the breakdown in the $I_{C}$-vs- $I_{B}$ characteristics occurs at reduced $I_{B}$ for increasing $V_{E C}$. Further increase of $V_{E C}$ beyond the breakdown in $I_{C}$ leads to a total quenching of the optical output intensity. This may be due to a combination of several effects. The increase in $V_{E C}$ will partly add to the band bending in the base-collector junction and thereby enhance a possible Franz-Keldysh absorption process (although as discussed in conjunction with Fig. 3, the dependency on $I_{B}$ counteracts this argument). Moreover, the increase in $I_{C}$ and $V_{E B}$ (which essentially replicates $I_{C}$ since $I_{E}=I_{C}+I_{B}$ with $I_{B}$ kept constant and $I_{E}$ controlled by $V_{E B}$; see Figs. 3 and 5) will result in device heating that will affect the optical gain, and the increase in $V_{E B}$ may, as discussed above, itself lead to a quenching of the emission.

The low optical collector offset voltage $\left(0.55 \mathrm{~V}\right.$ at $\left.I_{B t h}\right)$ yields a power consumption of only $1.95 \mathrm{~mW}$ (calculated as $I_{B} \cdot V_{E B}+I_{C} \cdot V_{E C}$ ) under lasing which is significantly lower than previous reports for T-VCSELs [2]. From Fig. 4 it is also noted that for large values of $V_{E C}$ (around $5 \mathrm{~V}$ ) there is a clear reduction in $I_{B t h}$. As indicated in the inset of Fig. 4, this is interpreted to be due to electron tunneling across the reversebiased base-collector junction at sufficiently high $V_{B C}=V_{E C^{-}}$ $V_{E B}$ (according to contribution 4 in Fig. 1).

Figure 5 shows $I_{C}, V_{E B}$ and $P_{\text {out }}$ as function of $I_{B}$ for a 4- $\mu \mathrm{m}$ device at different temperatures between 20 and $60^{\circ} \mathrm{C}$. Continuous-wave operation with the T-VCSEL in its active mode of operation is observed over the full temperature range. Notably, the temperature dependencies of $I_{B, B D}$ and $V_{E B}$ are quite significant and appear to be inconsistent with the expectations from a Franz-Keldysh-governed absorption mechanism. For instance, at $I_{B, B D}$ for the $60^{\circ} \mathrm{C}$ curve, $V_{E B}$ is larger (by $0.55 \mathrm{~V}$ ) and $P_{\text {out }}$ is lower (by $35 \%$ ) at $60^{\circ} \mathrm{C}$ as compared to the case of $20^{\circ} \mathrm{C}$. Still, the breakdown occurs at significantly lower current (2.2 rather than $5.3 \mathrm{~mA}$ ), which is inconsistent with the requirement on a high photon density in combination with a large band bending in the base-collector junction. Instead we suggest that the assumption of QW band filling would better match the observed breakdown characteristics. Saturation of the available states as $I_{E}$ is increased will lead to an increasing base transport factor 


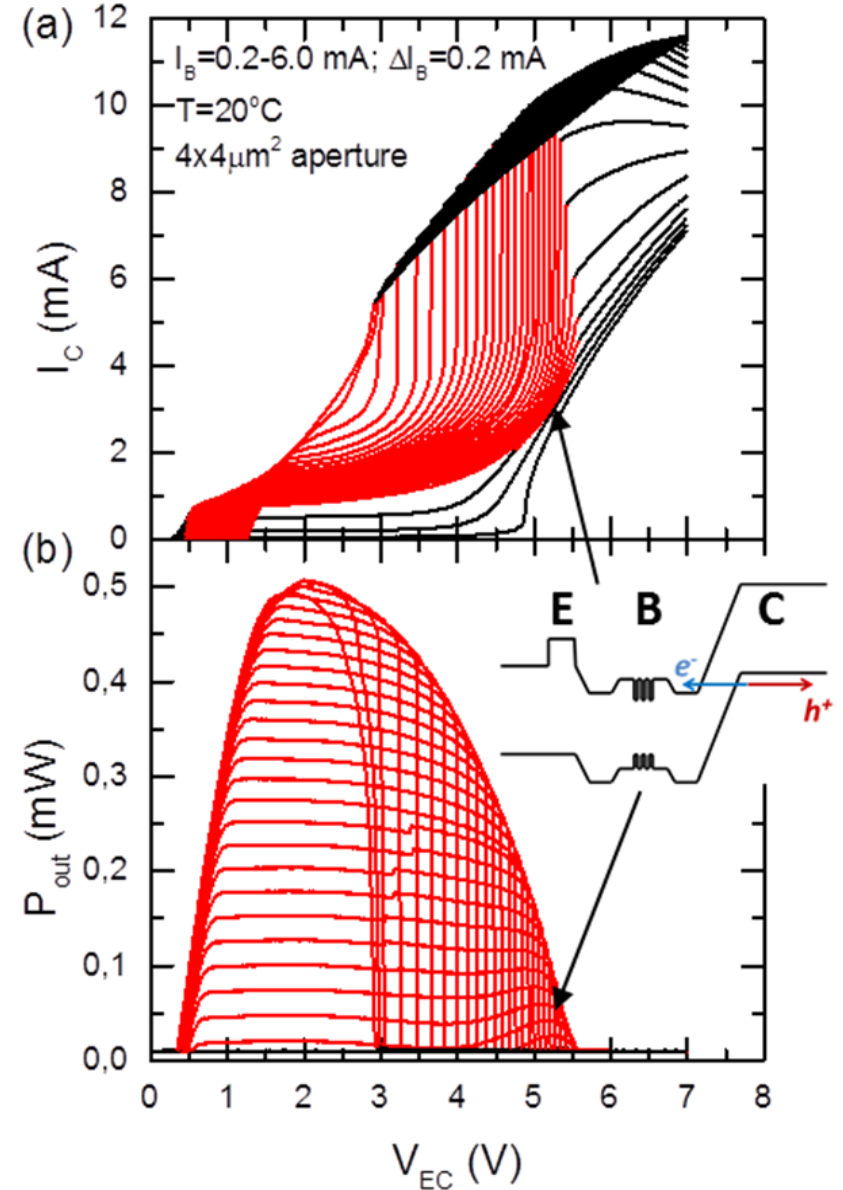

Fig. 4. Collector current and optical output power as function of emittercollector voltage at different base currents ranging between 0.2 and $6 \mathrm{~mA}$. Red line color indicates stimulated emission. A clear gain compression is observed at the onset of lasing. The inset shows a simplified band diagram corresponding to tunneling across the reverse-biased base-collector junction as reflected by a reduction of $I_{B t h}$ around $V_{E C}=5 \mathrm{~V}$.

( $\left.\alpha_{T}=I_{C} / I_{E h}\right)$ that increases $I_{C}$, and due to the corresponding reduction in recombination current, also increases $V_{E B}$ that in turn drives $I_{E}$ and thereby the breakdown in $I_{C}$. This also complies with the observed temperature dependency since an increase in temperature will lead to increased carrier leakage over the QWs [10], further increasing $I_{C}$.

\section{SUMMARY}

To summarize, we have demonstrated that pnp-type TVCSELs can be tuned for an extended range of active mode of transistor operation from a proper design of the base region. The fabricated devices operate continuous wave over a large temperature range up to at least $60^{\circ} \mathrm{C}$, and show optical output power and threshold base current in the $\mathrm{mW}$ and $\mathrm{mA}$ regimes, respectively, thereby approaching the performance of standard diode-type VCSELs. Finally, we discussed the mechanism of breakdown in $I_{C}$ in the limit of high $I_{B}$ and/or $V_{E C}$, and based on the temperature dependency of $I_{B, B D}, V_{E B}\left(I_{B}\right)$ and $P_{\text {out }}\left(I_{B}\right)$ we conclude that this most likely not is due to a Franz-Keldysh absorption process as previously suggested for npn-type TVCSELs but more likely to a QW band-filling effect. In

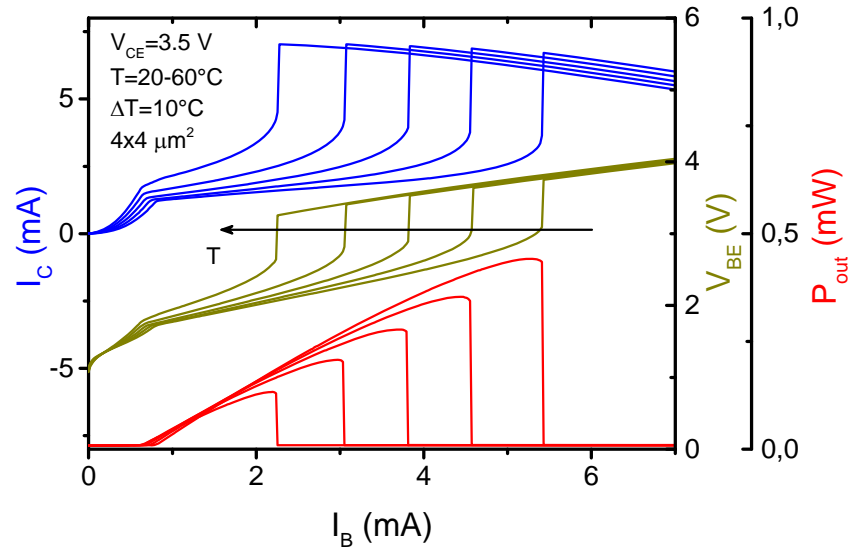

Fig. 5. Collector current, base-emitter voltage and output power as function of base current at different temperatures from 20 to $60^{\circ} \mathrm{C}$ as indicated. The aperture size is $4.4 \mu \mathrm{m}^{2}$. The more abrupt quenching of $P_{\text {out }}$ as compared to Fig. 3 reflects the higher resistance in the smaller device and thereby a steeper increase in $V_{E B}$.

addition, we note that for the quenching of the optical output power beyond breakdown, the measured LIV characteristics are not in agreement with expectations based on FranzKeldysh absorption.

\section{REFERENCES}

[1] H.W. Then, M. Feng and N. Holonyak, "The transistor laser: Theory and Experiment”, Proc. IEEE vol. 101, no. 10, pp. 2271-2298, October 2013

[2] M.K. Wu, M. Liu, R. Bambery, M. Feng, and N. Holonyak, "Low Power Operation of a Vertical Cavity Transistor Laser via the Reduction of Collector Offset Voltage”, IEEE Photonics Technol. Lett. Vol. 26, no. 10, pp. 1003- (2014)

[3] M.K. Wu, M. Feng, and N. Holonyak, "Surface emission vertical cavity transistor laser”, Photon. Technol. Lett. Vol. 24, no. 15, pp. 1346-1348 (2012)

[4] M.K. Wu, M. Feng, and N. Holonyak, "Voltage modulation of a vertical cavity transistor laser via intra-cavity photon-assisted tunneling”, Appl. Phys. Lett. Vol. 101, 081102-1-3 (2012)

[5] M. Liu, M.K. Wu, M. Feng and N. Holonyak, "Lateral feeding design and selective oxidation process in vertical cavity transistor laser, J. Appl. Phys. Vol. 114, pp. 163104-1-9 (2013)

[6] X. Yu, Y. Xiang, J. Berggren, T. Zabel, M. Hammar and N. Akram, W. Shi and L. Chrostowski, "Room-temperature operation of transistor vertical-cavity surface-emitting laser”, Electron. Lett., vol. 49, no. 3, pp. 208-209 (2013)

[7] Y. Xiang, X. Yu, J. Berggren, T. Zabel, M. Hammar and N. Akram, "Minority current distribution in InGaAs/GaAs transistor-verticalcavity surface-emitting laser” Appl. Phys. Lett. Vol. 102, pp. 191101-13 (2013)

[8] M.N. Akram, Y.Xiang, X. Yu, T. Zabel and M. Hammar, "Influence of base-region thickness on the performance of pnp transistor VCSEL", Optics Express Vol. 22, no. 2, pp. 27398-28414 (2014)

[9] A. James, G. Walter, M. Feng and N. Holonyak, "Photon-assisted breakdown, negative resistance, and switching in a quantum-well transistor laser”, Appl. Phys. Lett. 90, 152109 (2007)

[10] L.A. Coldren and S.W. Corzine, Diode Lasers and Photonic Integrated Circuits, Wiley, 1995, pp. 421-425 